\title{
FORMULATION OF FRACTIONAL DIFFERENTIAL EQUATIONS WITH INITIAL CONDITIONS
}

\author{
${ }^{1}$ Useni Paul Fatiye; ${ }^{1}$ Abegye S. Yakubu and ${ }^{1}$ Sunday Shammah Kpanja \\ ${ }^{1}$ Department of Mathematics \& Statistics, Isa Mustapha Agwai 1 Polytechnic, Lafia; Nigeria \\ Corresponding Author: Useni Paul Fatiye email: paul_useni@yahoo.com +2348036161732 \\ DOI: $10.31364 /$ SCIRJ/v9.i05.2021.P0521861 \\ http://dx.doi.org/10.31364/SCIRJ/v9.i05.2021.P0521861
}

\begin{abstract}
In this paper, we consider the Riemann-Liouville fractional derivative and the Caputo fractional derivative with particular reference to the initial conditions necessary for the formulation of fractional differential equations. We further determine the Riemann-Liouville and the Caputo fractional derivative of the function $f(x)=1$ stating their relationship. In conclusion, we use Laplace transform to establish a relationship between the Beta and the gamma functions.
\end{abstract}

Keywords: Riemann-Liouville, fractional derivative, Caputo, Laplace transform, fractional differential equations and integrals.

\subsection{Introduction}

In recent years it has turned out that many phenomena in engineering, Physics, Chemistry and other sciences can be described by models using Mathematical tools from fractional calculus $[6,7,8]$. In the $19^{\text {th }}$ century Abel formulated an integral equation which led to the development of fractional integro-differential operators by Liouville and Riemann [1, 2, 3, 4].

In the $20^{\text {th }}$ century, fractional derivatives found application in the study of viscoelasticity; diffusion and fluid dynamics problems amongst others with the advent of computers, numerical methods were developed for calculating the approximate solution to FDEs leading to an increase in their applications [5, 6].

The theory of derivatives and integrals of fractional order, some of the most prominent examples are given in a book of Oldham and Spanier [1]. In [ 9 ], the studied in details were description and assessment of numerical methods were described by fractional - order derivatives, integrals and differential equations which they provide an algorithm for calculating the Mittag-Leffler function which appears in solution to fractional - order differential equations.

2.0 Formulation of fractional differential equations.

By a first order ordinary fractional differential equation we will mean a fractional differential equation whose derivatives are all of order $<1$.

A first order fractional differential equation defined in terms of Riemann-Liouville derivatives of $f$ on $[a, b]$ integrate before differentiating $D J_{a}^{1-\alpha} f$

And the Caputo fractional derivative on $[a, b]$ is differentiable before integrating $J^{n+1} D^{n+1} f$, hence, for the RiemannLiouville derivatives will require the fractional integral $\lim _{t \rightarrow a} J_{a}^{1-\alpha} f(t)$ as an initial condition while a first order fractional ordinary differential equation defined in terms of Caputo derivatives will only require $f(a)$ as an initial condition.

$D J_{a}^{1-\alpha}=D\left\{\frac{\Gamma(1) x^{1-\alpha}}{\Gamma(2-\alpha)}\right\}=\frac{x^{-\alpha}}{\Gamma(1-\alpha)}$ From $J^{1-\alpha} x^{0}=\frac{\Gamma(0+1) x^{1-\alpha+0}}{\Gamma(1-\alpha+0+1)}=\frac{\Gamma(1) x^{1-\alpha}}{\Gamma(2-\alpha)}$ hence $J_{a}^{1-\alpha} D 1=J_{a}^{1-\alpha} 0=0$

www.sciri.org

(C) 2021, Scientific Research Journal

http://dx.doi.org/10.31364/SCIRJ/v9.i05.2021.P0521861

This publication is licensed under Creative Commons Attribution CC BY. 
The two derivatives are related by the formula $D_{a}^{\alpha}\left[f-T_{m-1}[f ; a]\right]=D_{a}^{\alpha} f$ where $T_{m-1}[f ; a]$ is the Taylor polynomial of degree $m^{-1}$ centered at $a$ and we shall call $D_{a}^{\alpha} f$ the Caputo fractional differential operators of advents, hence, $D_{a}^{\alpha} f=J_{a}^{m-\alpha} D^{m} f$.

3.0 Riemann-Liouville fractional integral of order $\alpha>0$, of $f(x)$ over the interval $[0, \infty)$.

The Riemann-Liouville fractional integral of the function $f$ of order $\alpha>0$, of $f(x)$ over the interval $[0, \infty)$ is given by $J_{0}^{\alpha} f(x)=\frac{1}{\Gamma(\alpha)} \int_{0}^{x} f(t)(x-t)^{\alpha-1} d t$ and the condition that $f$ must satisfy on this interval is given by $f \varepsilon L_{1}[0, \infty)$ which has to be integrated. Generally, we have that $J_{a}^{\alpha} f=\int_{a}^{x} f(t)(x-t)^{\alpha-1} d t, x \varepsilon[a, b]$.

Example: By the use of Laplace transforms show that $J^{\alpha} x^{\beta}=\frac{\Gamma(\beta+1)}{\Gamma(\alpha+\beta+1)} x^{\alpha+\beta}$ where $\beta>0$.

$J_{0}^{\alpha} x^{\beta}=\frac{1}{\Gamma(\alpha)} \int_{0}^{x} t^{\beta}(x-t)^{\alpha-1} d t$ Using convolution theory,

$\ell\left\{J_{0}^{\alpha} x^{\beta}\right\}=\frac{1}{\Gamma(\alpha)} \ell\left\{t^{\beta}\right\} \ell\left\{t^{\alpha-1}\right\}=\frac{1}{\Gamma(\alpha)} \frac{\Gamma(\beta+1) \Gamma(\alpha)}{S^{\beta+1} S^{\alpha}}=\frac{\Gamma(\beta+1)}{S^{\beta+1+\alpha}}$

Taking the inverse Laplace transform of both sides, we obtain

$J_{0}^{\alpha} f(x)=\Gamma(\beta+1) \frac{x^{\beta+\alpha}}{\Gamma(\beta+1+\alpha)}$

where $\beta>0$.

\subsection{Convergence of Riemann-Liouville fractional integral.}

Given that $f_{n}(x)=\frac{x^{2}}{1+1 / n}+\frac{1}{(2+x)^{n}}$ show that $\left\{f_{n}\right\}_{n=1}^{\infty}$

Converges uniformly to

$f(x)=x^{2}$ on $[0,1]$. Let $\varepsilon>0$ and consider $\left|f_{n}(x)-f(x)\right|=\left|\sum_{m=n+1}^{\infty} \frac{x^{m+1 / 2}}{m !}\right| \leq\left|\sum_{m=n+1}^{\infty} \frac{1}{m !}\right| \leq\left|\sum_{m=n+1}^{\infty} \frac{1}{(m-1)^{2}(m-2) !}\right|$

$\leq \frac{1}{n^{2}}\left|\sum_{m=n+1}^{\infty} \frac{1}{(m-2) !}\right| \leq \frac{1}{n^{2}} e^{1}$

Choose $n$ such that $\frac{e}{n^{2}}<\varepsilon,\left(\frac{e}{\varepsilon}\right)^{1 / 2}<n$.

Similarly, let $g_{n}(x)=\frac{x^{2}}{1+1 / n}$.

We shall confirm by fractional integration that $J_{0}^{1 / 2} g_{n}$ converges uniformly to $J_{0}^{1 / 2} g$ on the same initial interval. Where $J_{0}^{\alpha}$ denotes the Riemann-Liouville fractional integral operator of order $\alpha>0$ centered at 0 as follows.

www.scirj.org

(C) 2021, Scientific Research Journal

http://dx.doi.org/10.31364/SCIRJ/v9.i05.2021.P0521861

This publication is licensed under Creative Commons Attribution CC BY. 
If $g_{n} \rightarrow g$ uniformly on $[0,1]$ then $J^{\alpha} g_{n} \rightarrow J^{\alpha} g \therefore J_{0}^{\frac{1}{2}} g_{n}=J_{0}^{1 / 2} \sum_{i=0}^{n} \frac{x^{i+1 / 2}}{\Gamma(i+3 / 2)}$

$=\sum_{i=0}^{n} J_{0}^{1 / 2} \sum_{i=0}^{n} \frac{x^{i+1 / 2}}{\Gamma(i+3 / 2)}=\sum_{i=0}^{n} \frac{\Gamma(i+3 / 2) x^{i+1}}{\Gamma(i+3 / 2) \Gamma(i+3 / 2+1 / 2)}=\sum_{i=0}^{n} \frac{x^{i+1}}{\Gamma(i+2)}=\sum_{i=0}^{n} \frac{x^{i+1}}{(i+1) !}$

$=\left(\sum_{i=0}^{n+1} \frac{x^{i}}{i !}\right)-1$ as $n \rightarrow \infty$,

$J_{0}^{\frac{1}{2}} g_{n}(x)=e^{x}-1$

\section{0}

\section{Fractional differential equations or Laplace transform}

Considering the fractional differential equation of the type $D_{0}^{\alpha} y=-\lambda^{\alpha} y, y(0)=y_{0}$ and $0<\alpha<1$. First, we recognize that the Caputo fractional derivative can be written as a convolution equation

$$
\begin{aligned}
& D_{0}^{\alpha} y=\frac{1}{\Gamma(1-\alpha)} \int_{0}^{t} \frac{y^{\prime}(x)}{(t-x)^{\alpha}} d x \\
& D_{0}^{\alpha} y=\frac{1}{\Gamma(1-\alpha)} t^{-\alpha} * y^{\prime}(t)=-\lambda^{\alpha} y .
\end{aligned}
$$

Using the notation $\ell(y)=\bar{y}$, we apply the Laplace transform to (4) and the result is obtained as follows.

$$
\begin{aligned}
& \frac{1}{\Gamma(1-\alpha)} \ell\left\{t^{-\alpha} * y^{\prime}(t)\right\}=-\lambda^{\alpha} \ell\{y\} \\
& \frac{1}{\Gamma(1-\alpha)} \ell\left\{t^{-\alpha}\right\} \ell\left\{y^{\prime}(t)\right\}=-\lambda^{\alpha} \bar{y} \\
& \Rightarrow \frac{1}{S^{1-\alpha}}\left(S \bar{y}-y_{0}\right)=-\lambda^{\alpha} \bar{y} \\
& \left(S^{\alpha}+\lambda^{\alpha}\right)_{y}^{-}=y_{0} S^{\alpha-1}=-\bar{y}=\frac{y_{0} S^{\alpha-1}}{S^{\alpha}+\lambda^{\alpha}} \text { Taking the inverse Laplace transform, it gives } \\
& y(t)=y_{0} E_{\alpha}\left(-(\lambda t)^{\alpha}\right)
\end{aligned}
$$

\subsection{Fractional integration}

Suppose that $f(x)=(x-a)^{\beta}$, where $\beta>0$ and let $n>0$ then,

$$
J_{a}^{n} f(x)=\frac{\Gamma(\beta+1)}{\Gamma(n+\beta+1)}(x-a)^{n+\beta}
$$

From the definition of the integral, we have $J_{a}^{n} f(x)=\frac{1}{\Gamma(n)} \int_{a}^{x}(t-a)^{\beta}(x-t)^{n-1} d t$

Let $t=a+s(x-a)$ then $d t=(x-a) d s$ and substituting that into the integral gives 


$$
\begin{aligned}
& J_{a}^{n} f(x)=\frac{1}{\Gamma(n)} \int_{0}^{1}[s(x+a)]^{\beta}(x-a)^{n-1}\left(1-s^{n-1}\right)(x-a) d s \\
& J_{a}^{n} f(x)=\frac{(x-a)^{n+\beta}}{\Gamma(n)} \int_{0}^{1} s^{\beta}(1-s)^{n-1} d s \\
& \text { But } \int_{0}^{1} s^{\beta}(1-s)^{n-1} d s=\frac{\Gamma(n) \cdot \Gamma(\beta+1)}{\Gamma(n+\beta+1)} \text { hence } \\
& J_{a}^{n} f(x)=\frac{\Gamma(\beta+1)}{\Gamma(n+\beta+1)}(x-a)^{n+\beta}
\end{aligned}
$$

\subsection{Relationship between Beta function and gamma function}

Here we shall use the Laplace transform to establish the relationship between the Beta function and the gamma function as presented below:

Suppose that $\beta(z, \omega)=\frac{\Gamma(z) \Gamma(\omega)}{\Gamma(z+\omega)}$ we can show that the RHS is equal to the LHS as follows:

Given $\beta(z, \omega)=\int_{0}^{1} t^{z-1}(1-t)^{\omega-1} d t \quad$ using convolution theory,

$\beta(z, \omega)(x)=\int_{0}^{x} t^{z-1}(1-t)^{\omega-1} d t$ Taking Laplace transform of both sides

$\ell\{\beta(z, \omega)(x)\}=\ell\left\{t^{z-1}\right\} \ell\left\{t^{\omega-1}\right\}=\frac{\Gamma(z)}{s^{z}} \frac{\Gamma(\omega)}{s^{\omega}}=\frac{\Gamma(z) \Gamma(\omega)}{s^{z+\omega}}$

Taking inverse Laplace transform of both sides

$$
\begin{aligned}
& \ell^{-1}\{\beta(z, \omega)(x)\}=\ell^{-1}\left\{\frac{\Gamma(z) \Gamma(\omega)}{s^{z+\omega}}\right\} \\
& \beta(z, \omega)=\frac{\Gamma(z) \Gamma(\omega)}{\Gamma(z+\omega)} x^{z+\omega-1} \text { Let } x=1 \\
& \beta(z, \omega)=\frac{\Gamma(z) \Gamma(\omega)}{\Gamma(z+\omega)} \\
& \text { Hence, show that } \int_{0}^{a} \frac{d y}{\sqrt{a^{4}-y^{4}}}=\frac{\Gamma(1 / 4)^{2}}{4 a \sqrt{2 \pi}} \\
& \text { Suppose } \frac{y}{a}=t^{1 / 4}, \text { which gives } \\
& \int_{0}^{a} \frac{d y}{\sqrt{a^{4}-y^{4}}}=\frac{1}{a^{2}} \int_{0}^{a}\left(1-\frac{y^{4}}{a^{4}}\right)^{-\frac{1}{2}} d y \\
& =\frac{1}{a^{2}} \int_{0}^{1}(1-t)^{-\frac{1}{2}} \frac{a t^{-3 / 4}}{4} d t=\frac{1}{4 a} \int_{0}^{1}(1-t)^{\frac{1}{2}-1} t^{\frac{1}{4}-1} t
\end{aligned}
$$




$$
\begin{aligned}
& =\frac{1}{4 a} \beta\left(\frac{1}{2}, \frac{1}{4}\right)=\frac{1}{4 a} \frac{\Gamma\left(\frac{1}{2}\right) \Gamma\left(\frac{1}{4}\right)}{\Gamma\left(\frac{3}{4}\right)} \\
& \frac{1}{4 a} \frac{\Gamma(1 / 2) \Gamma(1 / 4)^{2}}{\Gamma(3 / 4) \Gamma(1 / 4)} \text { Recollecting that } \Gamma(1 / 2)=\sqrt{\pi} \text { and } \Gamma(3 / 4) \Gamma(1 / 4)=\frac{\pi}{\sin \frac{\pi}{4}}
\end{aligned}
$$

gives the result.

\subsection{Conclusion}

We conclude that the Riemann-Liouville derivative on $[a, b]$ is integrated before differentiating and the Caputo fractional derivative on $[a, b]$ is differentiated before integrating. It was also discovered that the Riemann-Liouville derivative will require the fractional integral of $\lim _{t \rightarrow a} J_{a}^{1-\alpha} f(t)$ require $f(a)$ as an initial condition.

Finally, we observed that in calculating the fractional differential equation, the Caputo fractional derivative will first be written as a Convolution equation and both Laplace transform and inverse Laplace transform can be apply to obtain the general solution.

\section{Acknowledgement}

The authors are so grateful to the reviewers whose suggestions and corrections help to improve and add value to the paper, and also thankful to our HOD, Mathematics and Statistics, Mr. Dansadiq Adams Ishaya for his encouragement and support.

\section{References}

[1] I. Podlubny, Fractional differential equations, Academic press, San Diego, 1999.

[2] S.G. Samko, A.A. Kilbas, O.J Marichev, Fractional Integrals and derivatives. Theory and Applications, Gordon and Breach, Yverdon, 1993.

[3] K.Dicthelm, N.J. Ford, A.D. Freed, A Predictor-corrector approach for the numerical solution of fractional differential equations, nonlinear Dynamics, 29(2002).

[4] K.Dicthelm, N.J. Ford, Analysis of fractional differential equations, J. Math.Anal. Appl. Pp 229-248; 265(2002).

[5] V. Daftardar-Gejji.S.Bhalekar, Chaos in fractional ordered Liu System, Comput. Math. Appl, pp 1117-1127, 59(2010).

[6] Kilbas A.A, Srivastava H.M and Trujillo J.J, Theory and Applications of fractional differential equations (Amsterdam Elsevier), 2006.

[7] Magin R.L, Fractional Calculus in Bioengineering (redding, CT. Begell House), 2006.

[8] Gorenflo R and Mainardi F, Fractional Calculus: Integral and differential equations of fractional order fractals and fractional calculus in continuum mechanics .ed a carpineri and F. mainardi (Wien:springer). 1997.

[9] K.Dicthelm, N.J. Ford, A.D. Freed,Yu.Luchko, Algorithms for the fractional calculus: A selection of numerical methods. Comput. Methods Appl. Mech, Engrg. Pp 743-773, 194(2005).

www.sciri.org

(C) 2021, Scientific Research Journal

http://dx.doi.org/10.31364/SCIRJ/v9.i05.2021.P0521861

This publication is licensed under Creative Commons Attribution CC BY. 